\title{
A new three-parameter exponential distribution with appli- cations in reliability and engineering
}

\author{
Maha A. Aldahlan ${ }^{\mathrm{a}, *}$, Ahmed Z. Afify ${ }^{\mathrm{b}}$ \\ ${ }^{a}$ Department of Statistics, College of Science, University of Jeddah, Jeddah, Saudi Arabia. \\ ${ }^{b}$ Department of Statistics, Mathematics and Insurance, Benha University, Egypt.
}

\begin{abstract}
We introduce a new three-parameter model called the odd inverse Pareto exponential distribution which extends the exponential distribution and provides constant, decreasing, increasing, decreasing-increasing, upside-down bathtub and bathtub failure rate shapes. Some of its mathematical properties are derived. The maximum likelihood method is used to estimate the model parameters. The proposed model provides better fits over some existing distributions by means of two real data sets.
\end{abstract}

Keywords: Exponential distribution, generating function, inverse Pareto-G family, maximum likelihood, order statistics. 2010 MSC: 62E10, 60E05.

(C)2020 All rights reserved.

\section{Introduction}

The exponential (Ex) distribution has been used extensively for analyzing lifetime data because it is analytically tractable and has the "lack of memory property". However, with a constant hazard shape, the Ex distribution is not able to fit data sets with different hazard shapes as increasing, decreasing, bathtub or upside down bathtub shaped (unimodal) failure rates, often encountered in reliability, engineering and biological studies. For many years, researchers have been developing various extensions and modified forms of the Ex distribution, for example: the exponentiated Ex (Gupta and Kundu, [8]) and beta Ex (Jones, [9]) distributions which can have monotone hazard rate shapes.

Several extensions of the Ex distribution have been proposed in the literature namely, the MarshallOlkin Ex distribution due to Marshall and Olkin [15], logistic Ex distribution due to Lan and Leemis [12], Nadarajah-Haghighi Ex distribution due to Nadarajah and Haghighi [16], modified Ex distribution due to Rasekhi et al. [18], odd exponentiated half-logistic Ex distribution due to Afify et al. [2], and extended odd Weibull exponential distribution due to Afify and Mohamed [4], among others.

In this paper, we propose and study a new three-parameter model called the odd inverse Paretoexponential (OIPEx) distribution. The proposed model has the advantage of being capable of modeling

\footnotetext{
${ }^{*}$ Corresponding author

Email addresses: maal-dahlan@uj.edu.sa (Maha A. Aldahlan), ahmed.afify@fcom.bu.edu.eg (Ahmed Z. Afify)

doi: $10.22436 /$ jnsa.013.05.03
}

Received: 2020-01-07 Revised: 2020-02-01 Accepted: 2020-02-05 
various shapes of aging and failure criteria, namely, constant, increasing, decreasing, bathtub or upsidedown bathtub. Accordingly, it can be used effectively to analyze lifetime data sets. It is noted that very few three-parameter distributions can give rise to the five main shapes of the hazard rate function (HRF). [6].

We construct the new model based on the odd inverse Pareto-G (OIP-G) family due to Aldahlan et al.

The cumulative distribution function (CDF) of the OIP-G family is given (for $x>0$ ) by

$$
\mathrm{F}(\mathrm{x} ; \alpha, \beta, \boldsymbol{\varphi})=\alpha \beta \int_{0}^{\frac{\mathrm{G}(x ; \boldsymbol{\varphi})}{1-\mathrm{G}(\mathrm{x} ; \boldsymbol{\varphi})}} \mathrm{t}^{\alpha-1}(\beta+\mathrm{t})^{-\alpha-1} \mathrm{dt}=\frac{\mathrm{G}(\mathrm{x} ; \boldsymbol{\varphi})^{\alpha}}{[1-\mathrm{G}(\mathrm{x} ; \boldsymbol{\varphi})]^{\alpha}}\left[\beta+\frac{\mathrm{G}(\mathrm{x} ; \boldsymbol{\varphi})}{1-\mathrm{G}(\mathrm{x} ; \boldsymbol{\varphi})}\right]^{-\alpha} .
$$

The corresponding probability density function (PDF) is given by

$$
f(x ; \alpha, \beta, \varphi)=\frac{\alpha \beta g(x ; \varphi) G(x ; \varphi)^{\alpha-1}}{[1-G(x ; \varphi)]^{\alpha+1}}\left[\beta+\frac{G(x ; \varphi)}{1-G(x ; \varphi)}\right]^{-\alpha-1},
$$

where $\mathrm{G}(x ; \varphi)$ is a baseline CDF with parameter vector $\varphi, g(x ; \varphi)=\mathrm{dG}(x ; \varphi) / \mathrm{d} x, \alpha>0$ and $\beta>0$ are two additional shape parameters. For $\beta=1$, the OIP-G family reduces to the exponentiated-G family (Gupta et al., [7]) . For $\alpha=1$, the OIP-G family becomes the Marshall-Olkin-G family (Marshall and Olkin, [15]). Clearly, for $\alpha=\beta=1$, we obtain the baseline model.

The HRF of the OIP-G family is given by

$$
\tau(x ; \alpha, \beta, \varphi)=\frac{\alpha \beta g(x ; \varphi) G(x ; \varphi)^{\alpha-1}\left[\beta+(1-\beta) G\left(x_{i} ; \varphi\right)\right]^{-1}}{\left[\beta+(1-\beta) G\left(x_{i} ; \varphi\right)\right]^{\alpha}-G(x ; \varphi)^{\alpha}} .
$$

The rest of the paper is outlined as follows. In Section 2, we define the OIPEx model and provide some plots for its PDF and HRF. In Section 3, we derive some mathematical properties of the OIPEx distribution. Estimation of the model parameters by maximum likelihood and a small simulation study are presented in Section 4. In Section 5, we prove empirically the flexibility of the new model using two applications to real data. Finally, some conclusions are presented in Section 6.

\section{The OIPEx distribution}

Consider the PDF and CDF (for $x>0) g(x)=\lambda \exp (-\lambda x)$ and $G(x)=1-\exp (-\lambda x)$, respectively, of the Ex distribution with positive parameters $\lambda$. Then, the PDF of the OIPEx model is given by

$$
f(x ; \alpha, \beta, \lambda)=\frac{\alpha \beta \lambda \exp (-\lambda x)[1-\exp (-\lambda x)]^{\alpha-1}}{[1-(1-\beta) \exp (-\lambda x)]^{\alpha+1}}, x>0, \alpha, \beta, \lambda>0 .
$$

The corresponding CDF of (2.1) is given by

$$
F(x ; \alpha, \beta, \lambda)=\frac{[1-\exp (-\lambda x)]^{\alpha}}{[1-(1-\beta) \exp (-\lambda x)]^{\alpha}}, x>0, \alpha, \beta, \lambda>0
$$

The HRF of the OIPEx model is given by

$$
\tau(x ; \alpha, \beta, \lambda)=\frac{\alpha \beta \lambda \exp (-\lambda x)[1-\exp (-\lambda x)]^{\alpha-1}[1-(1-\beta) \exp (-\lambda x)]^{-\alpha-1}}{1-[1-\exp (-\lambda x)]^{\alpha}[1-(1-\beta) \exp (-\lambda x)]^{-\alpha}} .
$$

Henceforth, a random variable with $\operatorname{PDF}(2.1)$ is denoted by $X \sim \operatorname{OIPEx}(\alpha, \beta, \lambda)$. Some special cases of the OIPEx distribution are: the exponentiated Ex (EEx) distribution (Gupta and Kundu, [8]) for $\beta=1$; the the Marshall-Olkin Ex distribution (Marshall and Olkin, [15]) for $\alpha=1$; the the Ex distribution for $\alpha=\beta=1$. The PDF and HRF plots of the OIPEx model are displayed in Figure 1. Figure 1 reveals that the PDF of the OIPEx can be reversed J-shape, concave down, symmetric, unimodal, left skewed or right skewed. The OIPEx HRF can be constant, decreasing, increasing, decreasing-increasing, upside down bathtub or bathtub failure rate shapes. 

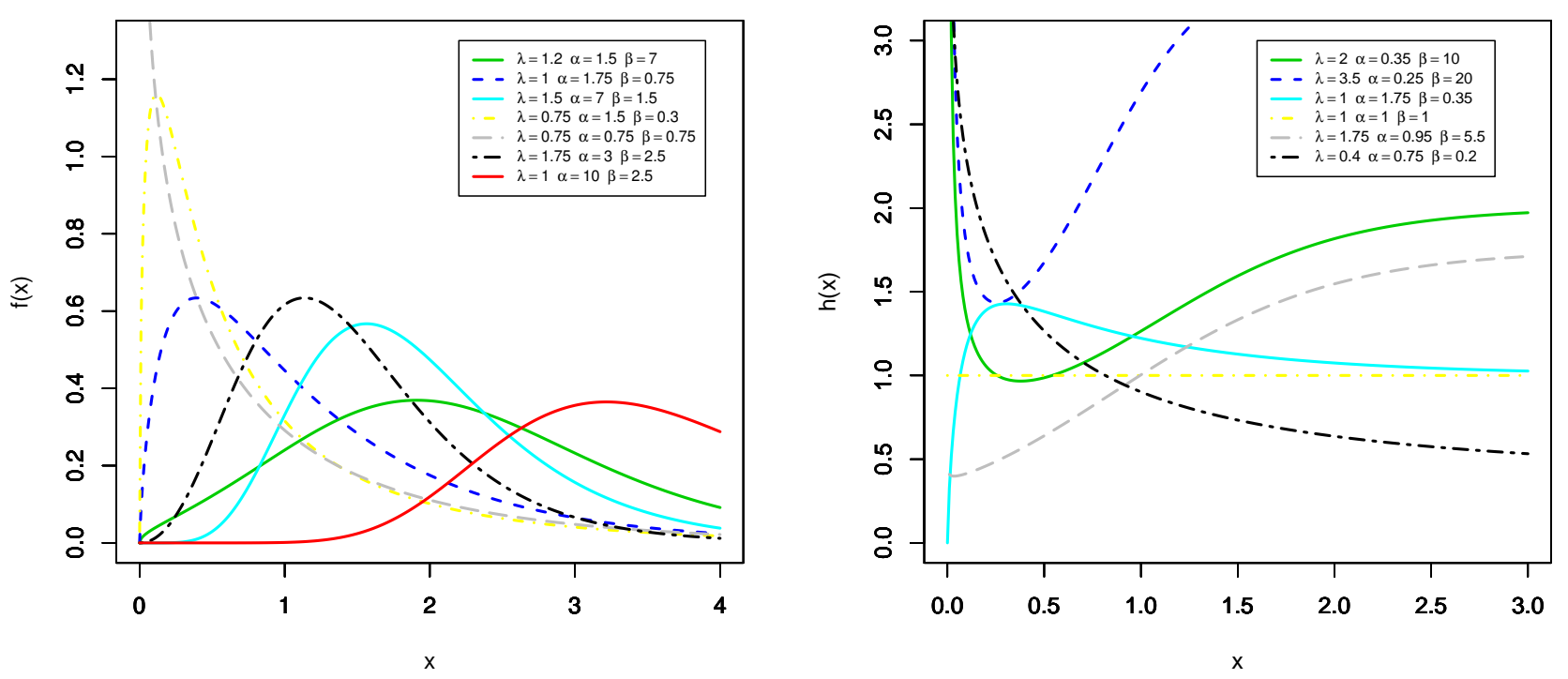

Figure 1: PDF and HRF plots of the OIPEx distribution for selected parameter values.

\section{Properties}

In this section, we provide some properties of the OIPEx distribution including quantile function (QF), moment generating function (MGF), ordinary and incomplete moments, mean residual life (MRL), mean inactivity time (MIT) and order statistics.

Hereafter, let $Z$ be a random variable having the Ex distribution, $g(z)=\lambda \exp (-\lambda z), \lambda, z>0$. Thus, the $r^{\text {th }}$ ordinary moment, $r^{\text {th }}$ incomplete moment, and MGF of $Z$ are given, respectively, by

$$
\mu_{r, Z}^{\prime}=\lambda^{-r} \Gamma(r+1), \quad \phi_{r, Z}(t)=\lambda^{-r} \gamma(r+1, \lambda t), \quad \text { and } M_{Z}(t)=\frac{\lambda}{\lambda-t}, t \neq 0,
$$

where $\Gamma(\cdot)$ and $\gamma(\cdot, \cdot)$ are the complete and lower incomplete gamma functions, respectively.

Remark 3.1. The PDF of the OIPEx distribution can be expressed as a mixture of Ex densities

$$
f(x)=\sum_{i=0}^{\infty} v_{i} g_{i+1}(x ;(i+1) \lambda)
$$

where $g_{i+1}(x ;(i+1) \lambda)=(i+1) \lambda \exp [-(i+1) \lambda x]$ is the Ex density with scale parameter $(i+1) \lambda$ and

$$
v_{i}=\sum_{k, j, i=0}^{\infty} \frac{\alpha(-1)^{j+i}}{(i+1) \beta^{k+\alpha}}\left(\begin{array}{c}
-\alpha-1 \\
k
\end{array}\right)\left(\begin{array}{c}
-k-\alpha-1 \\
j
\end{array}\right)\left(\begin{array}{c}
k+j+\alpha-1 \\
i
\end{array}\right) .
$$

Remark 3.1 reveals that several mathematical properties of the OIPEx distribution can be obtained directly from those properties of the Ex distribution.

Proof. Using equation (3.5) in Aldahlan et al. [6], the PDF of the OIPEx distribution can be expressed as

$$
f(x)=\sum_{k, j=0}^{\infty} d_{k, j} h_{k+j+\alpha}(x),
$$


where $h_{k+j+\alpha}(x)=(k+j+\alpha) \lambda \exp (-\lambda x)[1-\exp (-\lambda x)]^{k+j+\alpha-1}$ is the exponentiated Ex PDF with power parameter $(k+j+\alpha)$ and

$$
d_{k, j}=\frac{\alpha(-1)^{j}}{(k+j+\alpha) \beta^{k+\alpha}}\left(\begin{array}{c}
-\alpha-1 \\
k
\end{array}\right)\left(\begin{array}{c}
-k-\alpha-1 \\
j
\end{array}\right) .
$$

Using the generalized binomial series to $[1-\exp (-\lambda x)]^{k+j+\alpha-1}$, we can write equation (3.2) as

$$
f(x)=\sum_{k, j, i=0}^{\infty} \frac{\alpha(-1)^{j+i}}{\beta^{k+\alpha}}\left(\begin{array}{c}
-\alpha-1 \\
k
\end{array}\right)\left(\begin{array}{c}
-k-\alpha-1 \\
j
\end{array}\right)\left(\begin{array}{c}
k+j+\alpha-1 \\
i
\end{array}\right) \lambda \exp (-(i+1) \lambda x) .
$$

Or equivalently, we can write

$$
f(x)=\sum_{i=0}^{\infty} v_{i} g_{i+1}(x ;(i+1) \lambda)
$$

3.1. Quantile and generating functions

The QF of the OIPEx distribution follows, by inverting (2.2), as

$$
x_{\mathfrak{u}}=Q(u)=\frac{-1}{\lambda} \log \left(1-\frac{\beta u^{\frac{1}{\alpha}}}{1-(1-\beta) u^{\frac{1}{\alpha}}}\right) \text {. }
$$

Based on (3.3), we can obtain a random sample of size $n$ from (2.2), as $X_{i}=Q\left(U_{i}\right)$, where $U_{i} \sim \operatorname{Uniform}(0,1)$, $i=1,2, \ldots, n$.

Using (3.1) and the MGF of $Z$, the MGF of the OIPEx model can be rewritten as

$$
M_{X}(t)=\sum_{i=0}^{\infty} v_{i} \frac{(i+1) \lambda}{(i+1) \lambda-t}
$$

\subsection{Ordinary and incomplete moments}

The $s^{\text {th }}$ ordinary moment of $X$ follows from equation (3.1) as

$$
\mu_{s}^{\prime}=\sum_{i=0}^{\infty} v_{i}[(i+1) \lambda]^{-s} \Gamma(s+1) .
$$

The mean, variance, skewness and kurtosis of the OIPEx distribution are given in Table 1. The values of these measures are computed numerically for $\lambda=1$ and some selected values of $\alpha$ and $\beta$ using the $R$ software. Table 1 shows that the skewness of the OIPEx distribution can range in the interval $(0.90,3.4)$, whereas the spread for the OIPEx kurtosis is much larger ranging from 4.8 to 20.4.

Further, the $s^{\text {th }}$ incomplete moment of $X$ can be rewritten as

$$
\phi_{s}(t)=\sum_{i=0}^{\infty} v_{i}[(i+1) \lambda]^{-s} \gamma(s+1,(i+1) \lambda t) .
$$

The first incomplete moment follows from the above equation with $s=1$ as

$$
\phi_{1}(t)=\sum_{i=0}^{\infty} v_{i} \frac{\gamma(2,(i+1) \lambda t)}{[(i+1) \lambda]} .
$$


Table 1: Mean, variance, skewness and kurtosis of the OIPEx model for $\lambda=1$.

\begin{tabular}{|c|c|c|c|c|c|}
\hline$\alpha$ & $\beta$ & Mean & Variance & Skewness & Kurtosis \\
\hline 0.5 & 0.5 & 0.4134 & 0.4477 & 3.4198 & 20.404 \\
& 1.5 & 0.7600 & 0.9064 & 2.2620 & 10.326 \\
& 2.5 & 0.9769 & 1.1992 & 1.8672 & 7.8668 \\
& 5 & 1.3302 & 1.6696 & 1.4297 & 5.7148 \\
\hline 1.5 & 0.5 & 0.9063 & 0.8351 & 2.2451 & 10.671 \\
& 1.5 & 1.5374 & 1.3502 & 1.5127 & 6.5866 \\
& 2.5 & 1.8986 & 1.5914 & 1.2648 & 5.6093 \\
& 5 & 2.4463 & 1.8857 & 0.9993 & 4.8034 \\
\hline 2.5 & 0.5 & 1.2254 & 1.0221 & 1.9096 & 8.6525 \\
& 1.5 & 1.9825 & 1.4735 & 1.3464 & 6.0027 \\
& 2.5 & 2.3953 & 1.6497 & 1.1669 & 5.3933 \\
& 5 & 3.0006 & 1.8358 & 0.9881 & 4.9120 \\
\hline 5 & 0.5 & 1.7375 & 1.2432 & 1.5952 & 7.0798 \\
& 1.5 & 2.6303 & 1.5658 & 1.2302 & 5.6508 \\
& 2.5 & 3.0894 & 1.6655 & 1.1275 & 5.3409 \\
& 5 & 3.7399 & 1.7556 & 1.0363 & 5.1041 \\
\hline
\end{tabular}

\subsection{Mean residual life and mean inactivity time}

The life expectancy at age $t$ or MRL of $X$ is defined by

$$
m_{X}(t)=\left[1-\phi_{1}(t)\right] / \bar{F}(t)-t, t>0,
$$

where $\bar{F}($.$) is the survival function.$

By using (3.4) in equation (3.5), the MRL of $X$ can be determined as

$$
m_{X}(t)=\frac{1}{\bar{F}(t)} \sum_{i=0}^{\infty} v_{i} \frac{\gamma(2,(i+1) \lambda t)}{(i+1) \lambda}-t
$$

The MIT of $X$ is defined by

$$
m_{X}^{\prime}(t)=t-\left[\phi_{1}(t) / F(t)\right], t>0 .
$$

By inserting (3.4) in the last equation, the MIT of $X$ follows as

$$
m_{X}^{\prime}(t)=t-\frac{1}{F(t)} \sum_{i=0}^{\infty} v_{i} \frac{\gamma(2,(i+1) \lambda t)}{(i+1) \lambda} .
$$

\subsection{Order statistics}

Order statistics are important in several areas of statistical theory and practice. Let $X_{1}, \ldots, X_{n}$ be a random sample from the OIPEx distribution. The PDF of $X_{i: n}$ can be written as

$$
f_{i: n}(x)=\frac{f(x)}{B(i, n-i+1)} \sum_{j=0}^{n-i}(-1)^{j}\left(\begin{array}{c}
n-i \\
j
\end{array}\right) F(x)^{j+i-1},
$$

where $\mathrm{B}(\cdot, \cdot)$ is the beta function. Based on equations (2.1) and (2.2), and after some algebra, we can write

$$
f(x) F(x)^{j+i-1}=\alpha \sum_{k, l=0}^{\infty} \frac{(-1)^{l}}{\beta^{k+\alpha(j+i)}}\left(\begin{array}{c}
-\alpha(j+i)-1 \\
k
\end{array}\right)\left(\begin{array}{c}
-k-\alpha(j+i)-1 \\
l
\end{array}\right)
$$




$$
\times \lambda \exp (-\lambda x)[1-\exp (-\lambda x)]^{k+l+\alpha(j+i)-1} .
$$

Using the binomial expansion to $[1-\exp (-\lambda x)]^{k+l+\alpha(j+i)-1}$, the above equation reduces to

$$
\begin{aligned}
f(x) F(x)^{j+i-1}= & \alpha \sum_{k, l, p=0}^{\infty} \frac{(-1)^{l+p}}{\beta^{k+\alpha(j+i)}}\left(\begin{array}{c}
-\alpha(j+i)-1 \\
k
\end{array}\right)\left(\begin{array}{c}
-k-\alpha(j+i)-1 \\
l
\end{array}\right) \\
& \times\left(\begin{array}{c}
k+l+\alpha(j+i)-1 \\
p
\end{array}\right) \lambda \exp (-(p+1) \lambda x) .
\end{aligned}
$$

Inserting the last equation in (3.6), the PDF of $X_{i: n}$ follows as

$$
f_{i: n}(x)=\sum_{p=0}^{\infty} m_{p} g_{p+1}(x ;(p+1) \lambda),
$$

where $g_{p+1}(x ;(p+1) \lambda)$ is the Ex density with scale parameter $(p+1) \lambda$ and

$$
\begin{aligned}
m_{p}= & \alpha \sum_{k, l=0}^{\infty} \sum_{j=0}^{n-i} \frac{(-1)^{j+l+p} \beta^{-k-\alpha(j+i)}}{(p+1) B(i, n-i+1)}\left(\begin{array}{c}
n-i \\
j
\end{array}\right)\left(\begin{array}{c}
-\alpha(j+i)-1 \\
k
\end{array}\right) \\
& \times\left(\begin{array}{c}
-k-\alpha(j+i)-1 \\
l
\end{array}\right)\left(\begin{array}{c}
k+l+\alpha(j+i)-1 \\
p
\end{array}\right) .
\end{aligned}
$$

Hence, the PDF of the OIPEx order statistics is a linear combination of Ex densities. Based on equation (3.7), the $q^{\text {th }}$ moment of $X_{i: n}$ can be expressed as

$$
E\left(X_{i: n}^{q}\right)=\sum_{p=0}^{\infty} m_{p}[(p+1) \lambda]^{-q} \Gamma(q+1) .
$$

\section{Estimation and simulation}

Let $X_{1}, \ldots, X_{n}$ be a random sample from the OIPEx distribution with parameters $\alpha, \beta$ and $\lambda$. Let $\varphi=(\lambda, \alpha, \beta)^{\top}$ be the $p \times 1$ parameter vector. Then, the log-likelihood function for $\varphi$ is given by

$$
\begin{aligned}
\ell(\varphi)= & n \log (\alpha \beta \lambda)-\lambda \sum_{i=1}^{n} x_{i}+(\alpha-1) \sum_{i=1}^{n} \log \left(1-\exp \left(-\lambda x_{i}\right)\right) \\
& -(\alpha+1) \sum_{i=1}^{n} \log \left(1-(1-\beta) \exp \left(-\lambda x_{i}\right)\right)
\end{aligned}
$$

The score vector components, $\mathbf{U}(\varphi)=\frac{\partial \ell}{\partial \varphi}=\left(\mathrm{U}_{\lambda}, \mathrm{U}_{\alpha}, \mathrm{U}_{\beta}\right)^{\top}$, are given by

and

$$
\begin{aligned}
& \mathrm{u}_{\lambda}=\frac{n}{\lambda}-\sum_{i=1}^{n} x_{i}+(\alpha-1) \sum_{i=1}^{n} \frac{x_{i} \exp \left(-\lambda x_{i}\right)}{1-\exp \left(-\lambda x_{i}\right)}-(\alpha+1) \sum_{i=1}^{n} \frac{(1-\beta) x_{i} \exp \left(-\lambda x_{i}\right)}{1-(1-\beta) \exp \left(-\lambda x_{i}\right)^{\prime}} \\
& \mathrm{u}_{\alpha}=\frac{n}{\alpha}+\sum_{i=1}^{n} \log \left(1-\exp \left(-\lambda x_{i}\right)\right)-\sum_{i=1}^{n} \log \left(1-(1-\beta) \exp \left(-\lambda x_{i}\right)\right),
\end{aligned}
$$

$$
\mathrm{u}_{\beta}=\frac{\mathrm{n}}{\beta}-(\alpha+1) \sum_{i=1}^{\mathrm{n}} \frac{\exp \left(-\lambda x_{i}\right)}{1-(1-\beta) \exp \left(-\lambda x_{i}\right)}
$$


Setting the nonlinear system of equations $\mathrm{U}_{\lambda}=\mathrm{U}_{\alpha}=\mathrm{U}_{\beta}=0$ and solving them simultaneously yields the MLE $\widehat{\varphi}=(\widehat{\lambda}, \widehat{\alpha}, \widehat{\beta})^{\top}$. For doing this, it is usually more convenient to adopt nonlinear optimization methods such as the quasi-Newton algorithm to maximize $\ell$ numerically. For interval estimation of the parameters, we obtain the $p \times p$ observed information matrix $J(\varphi)=\left\{\frac{\partial^{2} \ell}{\partial r \partial s}\right\}$ (for $\left.r, s=\lambda, \alpha, \beta\right)$, whose elements can be computed numerically.

Now, we perform a small Monte Carlo simulation study to assess the performance of the ML estimators (MLEs) of the unknown parameters for the OIPEx distribution. The performance of the MLEs is evaluated in terms of their average values and mean squared errors (MSEs). The Mathcad program is used to generate 1000 samples of the OIPEx distribution for different sample sizes, $n=(50,100,150)$, and for different parameters combinations, $\lambda=(0.5,1.0), \alpha=(0.35,0.5,0.75,1.5)$ and $\beta=(0.25,0.35,0.5$, 1.0, 1.5, 3.5). The average values of estimates and MSEs are provided in Table 2. We noted, from Table 2, that the MSE decreases as the sample size increases. Thus, the MLE method works very well to estimate the model parameters of the OIPEx distribution.

Table 2: Average values of the estimates and the corresponding MSEs for the OIPEx distribution.

\begin{tabular}{|c|c|c|c|c|c|c|c|c|c|}
\hline $\mathrm{n}$ & \multicolumn{2}{|c}{ Parameters } & \multicolumn{2}{c}{ Average Estimates } & \multicolumn{3}{c|}{ MSEs } \\
\hline & $\lambda$ & $\alpha$ & $\beta$ & $\lambda$ & $\alpha$ & $\beta$ & $\lambda$ & $\alpha$ & $\beta$ \\
\hline 50 & 0.50 & 0.35 & 0.25 & 0.588 & 0.369 & 0.311 & 0.334 & 0.007 & 0.223 \\
100 & & & & 0.500 & 0.364 & 0.250 & 0.133 & 0.003 & 0.058 \\
150 & & & & 0.425 & 0.361 & 0.207 & 0.077 & 0.002 & 0.026 \\
\hline 50 & 0.50 & 0.35 & 0.35 & 0.614 & 0.368 & 0.479 & 0.240 & 0.006 & 0.342 \\
100 & & & & 0.505 & 0.360 & 0.368 & 0.097 & 0.002 & 0.115 \\
150 & & & & 0.471 & 0.361 & 0.329 & 0.071 & 0.002 & 0.064 \\
\hline 50 & 1.00 & 0.50 & 0.50 & 1.090 & 0.536 & 0.693 & 0.469 & 0.019 & 0.643 \\
100 & & & & 0.959 & 0.527 & 0.518 & 0.206 & 0.009 & 0.152 \\
150 & & & & 0.927 & 0.517 & 0.499 & 0.172 & 0.006 & 0.109 \\
\hline 50 & 1.00 & 0.50 & 1.00 & 1.113 & 0.536 & 1.364 & 0.204 & 0.020 & 1.760 \\
100 & & & & 1.037 & 0.521 & 1.100 & 0.082 & 0.008 & 0.391 \\
150 & & & & 0.999 & 0.516 & 1.030 & 0.048 & 0.005 & 0.236 \\
\hline 50 & 1.00 & 0.75 & 1.50 & 0.839 & 0.800 & 1.505 & 0.285 & 1.550 & 1.849 \\
100 & & & & 0.828 & 0.776 & 1.399 & 0.234 & 0.938 & 1.242 \\
150 & & & & 0.868 & 0.775 & 1.441 & 0.181 & 0.543 & 0.963 \\
\hline 50 & 1.00 & 1.50 & 3.50 & 1.004 & 2.050 & 3.836 & 0.035 & 0.919 & 1.049 \\
100 & & & & 0.995 & 1.761 & 3.543 & 0.018 & 0.526 & 0.562 \\
150 & & & & 0.991 & 1.664 & 3.474 & 0.011 & 0.149 & 0.214 \\
\hline
\end{tabular}

\section{Two applications}

We illustrate the flexibility of the OIPEx model using to real lifetime data sets. The first data set contains 40 observations and represents time to failure $\left(10^{3} \mathrm{~h}\right)$ of turbocharger of one type of engine (Xu et al., [19]). These data were analyzed by Afify et al. [1] and Nassar et al. [17]. The second data set consists of $n=74$ observations and represents the gauge lengths of $20 \mathrm{~mm}$ (Kundu and Raqab, [8]). These data were analyzed by Afify et al. [2]. The OIPEx distribution will be compared with some competitive models namely: the exponentiated exponential (EEx) due to Gupta and Kundu [8], beta exponential (BEx) due to Jones [9], transmuted generalized exponential (TGEx) due to Khan et al. [10], Kumaraswamy transmuted exponential (KTEx) due to Afify et al. [3], alpha power exponential (APEx) due to Mahdavi and Kundu [13], Marshall-Olkin logistic-exponential (MOLEx) due to Mansoor et al. [14], gamma (Ga), and Ex distributions whose PDFs are given by

$$
\operatorname{EEx}: f(x)=\alpha \lambda \exp (-\lambda x)[1-\exp (-\lambda x)]^{\alpha-1}
$$




$$
\begin{aligned}
& B E x: f(x)=\frac{\lambda}{B(a, b)} \exp (-b \lambda x)[1-\exp (-\lambda x)]^{a-1}, \\
& \text { TGEx :f }(x)=\alpha \lambda \exp (-\lambda x)[1-\exp (-\lambda x)]^{\alpha-1}\left\{1+\theta-2 \theta[1-\exp (-\lambda x)]^{\alpha}\right\} \text {, } \\
& \text { KTEx }: f(x)=\frac{a b \lambda \exp (-\lambda x)[1-\theta+2 \theta \exp (-\lambda x)]}{\{[1-\exp (-\lambda x)][1-\theta \exp (-\lambda x)]\}^{1-a}} \\
& \times\left(1-\{[1-\exp (-\lambda x)][1+\theta \exp (-\lambda x)]\}^{a}\right)^{b-1}, \\
& \text { APEx }: f(x)=\frac{\log (\alpha) \lambda \exp (-\lambda x)}{(\alpha-1)} \alpha^{1-\exp (-\lambda x)}, \alpha>0, \alpha \neq 1, \\
& \text { MOLEx :f }(x)=\alpha \theta \lambda \exp (\lambda x)[\exp (\lambda x)-1]^{-\alpha-1}\left\{1+\theta[\exp (\lambda x)-1]^{-\alpha}\right\}^{-2} \text {, } \\
& \text { Ga }: f(x)=\frac{b^{-a}}{\Gamma(a)} x^{a-1} \exp (-x / b) .
\end{aligned}
$$

The parameters of the above densities are all positive real numbers except for the KTEx and TGEx distributions for which $|\theta| \leqslant 1$.

The fitted distributions are compared using some goodness-of-fit criteria including the $-\widehat{\ell}$ (where $\widehat{\ell}$ is the maximized log-likelihood), CVM (Cramér-Von Mises), AD (Anderson-Darling) and KS (Kolmogorov Smirnov with its p-value (PV)) statistics.

The values of $-\widehat{\ell}, C V M, A D, K S, P V$, MLEs and their standard errors (SEs) (in parentheses) for both data sets are listed in Tables 3 and 4. The figures in these tables show that the OIPEx distribution has the lowest values for all goodness-of-fit statistics among all fitted distributions. So, it can be chosen as the

\begin{tabular}{|c|c|c|c|c|c|c|c|c|}
\hline Distribution & $-\hat{\ell}$ & CVM & $A D$ & KS & \multicolumn{4}{|c|}{ Estimates } \\
\hline $\begin{array}{l}\text { OIPEx } \\
(\alpha, \beta, \lambda)\end{array}$ & 80.870 & 0.0516 & 0.3260 & $\begin{array}{l}0.0937 \\
(0.8732)\end{array}$ & $\begin{array}{l}0.1899 \\
(0.0324)\end{array}$ & $\begin{array}{l}581876500 \\
(150.3)\end{array}$ & $\begin{array}{l}2.4253 \\
(0.0694)\end{array}$ & \\
\hline $\begin{array}{l}\text { KTEx } \\
(a, b, \lambda, \theta)\end{array}$ & 86.235 & 0.0725 & 0.5422 & $\begin{array}{l}0.1059 \\
(0.7605)\end{array}$ & $\begin{array}{l}2.9155 \\
(1.0753)\end{array}$ & $\begin{array}{l}2145.5 \\
(5616.4)\end{array}$ & $\begin{array}{l}0.0334 \\
(0.0241)\end{array}$ & $\begin{array}{l}-0.8222 \\
(0.3080)\end{array}$ \\
\hline $\begin{array}{l}\text { MOLEx } \\
(\alpha, \theta, \lambda)\end{array}$ & 86.598 & 0.0853 & 0.6136 & $\begin{array}{l}0.0910 \\
(0.8944)\end{array}$ & $\begin{array}{l}0.9174 \\
(0.9091)\end{array}$ & $\begin{array}{l}290.353 \\
(246.807)\end{array}$ & $\begin{array}{l}0.9654 \\
(0.9878)\end{array}$ & \\
\hline $\begin{array}{l}\mathrm{Ga} \\
(\mathrm{a}, \mathrm{b})\end{array}$ & 89.410 & 0.2052 & 1.3616 & $\begin{array}{l}0.1277 \\
(0.5311)\end{array}$ & $\begin{array}{l}7.7227 \\
(1.6908)\end{array}$ & $\begin{array}{l}1.2351 \\
(0.2794)\end{array}$ & & \\
\hline $\begin{array}{l}\text { BEx } \\
(a, b, \lambda)\end{array}$ & 90.417 & 0.2054 & 1.3626 & $\begin{array}{l}0.1283 \\
(0.5252)\end{array}$ & $\begin{array}{l}7.7267 \\
(1.6926)\end{array}$ & $\begin{array}{l}54.659 \\
(56.135)\end{array}$ & $\begin{array}{l}0.0213 \\
(0.0203)\end{array}$ & \\
\hline $\begin{array}{l}\text { APEx } \\
(\alpha, \lambda)\end{array}$ & 91.283 & 0.2187 & 1.4392 & $\begin{array}{l}0.1640 \\
(0.2318)\end{array}$ & $\begin{array}{l}20457 \\
(12390)\end{array}$ & $\begin{array}{l}0.4460 \\
(0.0321)\end{array}$ & & \\
\hline $\begin{array}{l}\text { TGEx } \\
(\alpha, \lambda, \theta)\end{array}$ & 91.670 & 0.2333 & 1.5194 & $\begin{array}{l}0.1448 \\
(0.3707)\end{array}$ & $\begin{array}{l}8.5465 \\
(3.1707)\end{array}$ & $\begin{array}{l}0.5000 \\
(0.0621)\end{array}$ & $\begin{array}{l}-0.6507 \\
(0.2560)\end{array}$ & \\
\hline $\begin{array}{l}\text { EEx } \\
(\alpha, \lambda)\end{array}$ & 92.142 & 0.2757 & 1.7600 & $\begin{array}{l}0.1541 \\
(0.2975)\end{array}$ & $\begin{array}{l}9.5142 \\
(2.8959)\end{array}$ & $\begin{array}{l}0.4498 \\
(0.0577)\end{array}$ & & \\
\hline $\begin{array}{l}\text { Ex } \\
(\lambda)\end{array}$ & 114.31 & 0.2065 & 1.3689 & $\begin{array}{l}0.3631 \\
(0.000)\end{array}$ & $\begin{array}{l}0.1599 \\
(0.0252)\end{array}$ & & & \\
\hline
\end{tabular}
best model to fit both data sets.

The plots of the fitted PDF of the OIPEx model and other fitted PDFs are displayed in Figures 2 and 3 , respectively. The corresponding probability probability (PP) plots, for both data sets, are displayed in Figures 4 and 5, respectively. These plots reveal that the OIPEx distribution provides the best fits to these data and it can be considered a very competitive model to other distributions with positive support.

Table 3: The goodness-of-fit statistics and estimates (PV and SEs in parentheses) for time to failure data. 

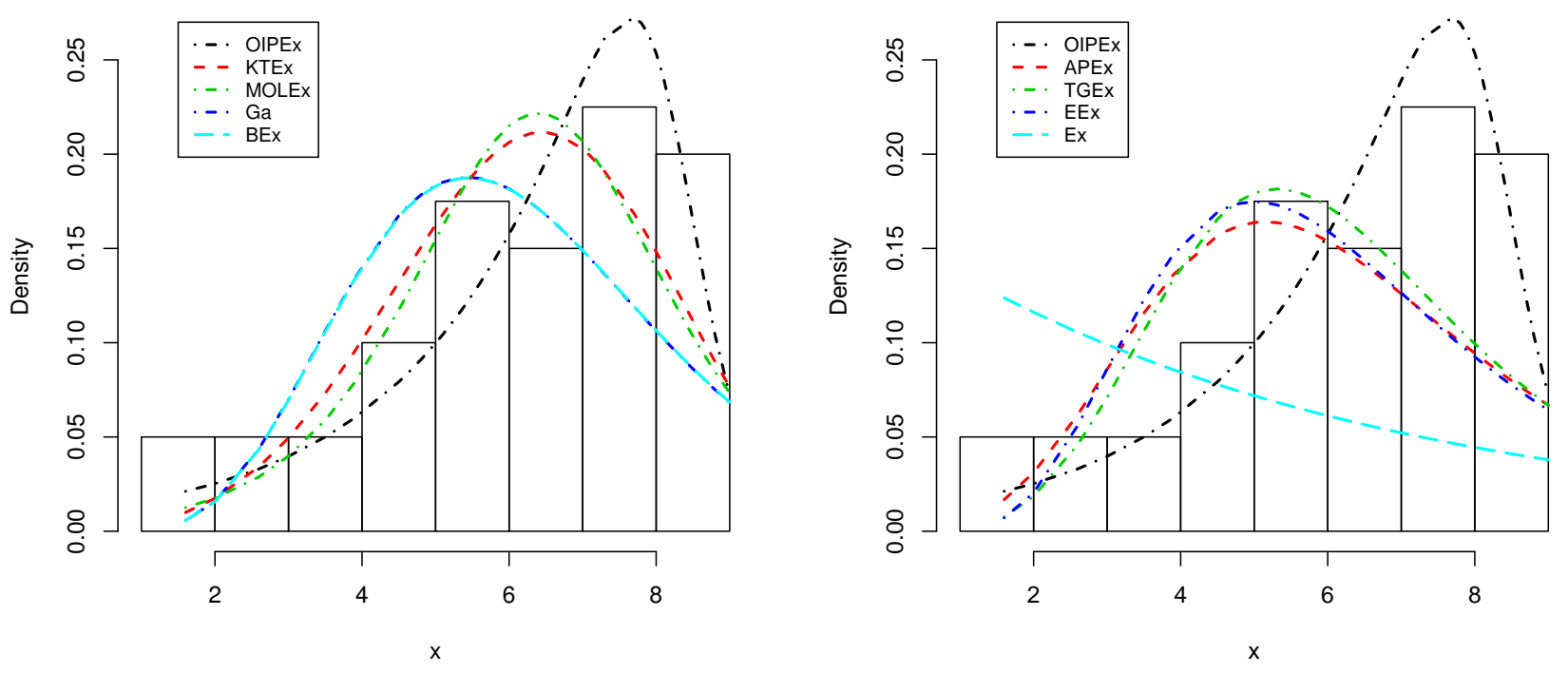

Figure 2: The fitted OIPEx PDF and other fitted PDFs for first data set.
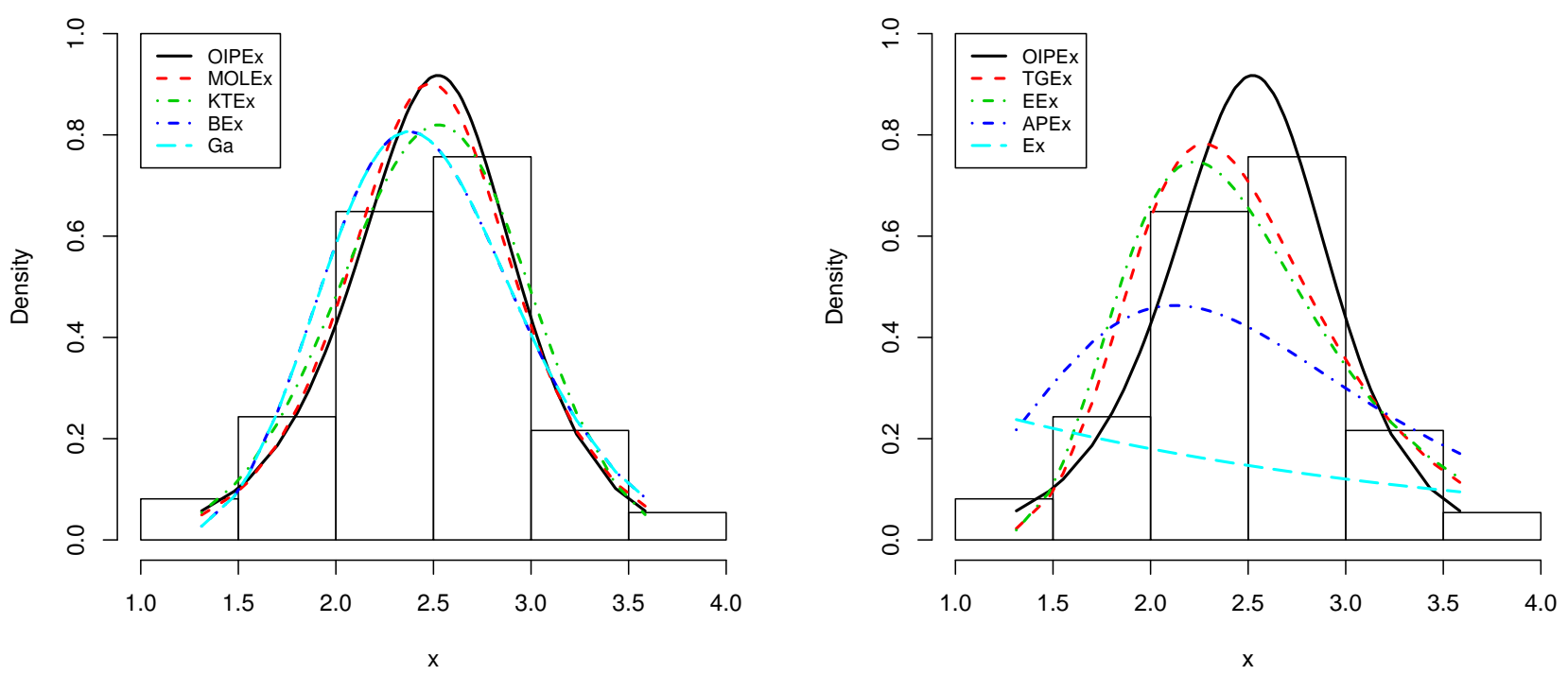

Figure 3: The fitted OIPEx PDF and other fitted PDFs for first data set. 
Table 4: The goodness-of-fit statistics and estimates (PV and SEs in parentheses) for gauge lengths data.

\begin{tabular}{|c|c|c|c|c|c|c|c|c|}
\hline Distribution & $-\widehat{l}$ & CVM & AD & KS & \multicolumn{4}{|c|}{ Estimates } \\
\hline $\begin{array}{l}\text { OIPEx } \\
(\alpha, \beta, \lambda)\end{array}$ & 51.835 & 0.0368 & 0.2355 & $\begin{array}{l}0.0591 \\
(0.9577)\end{array}$ & $\begin{array}{l}0.7950 \\
(0.2023)\end{array}$ & $\begin{array}{l}27297 \\
(34546)\end{array}$ & $\begin{array}{l}3.9576 \\
(0.3913)\end{array}$ & \\
\hline $\begin{array}{l}\text { MOLEx } \\
(\alpha, \theta, \lambda)\end{array}$ & 54.923 & 0.0451 & 0.2770 & $\begin{array}{l}0.0593 \\
(0.9569)\end{array}$ & $\begin{array}{l}1.8250 \\
(1.9746)\end{array}$ & $\begin{array}{l}7243.76 \\
(7213.1)\end{array}$ & $\begin{array}{l}1.9614 \\
(2.2110)\end{array}$ & \\
\hline $\begin{array}{l}\text { KTEx } \\
(a, b, \lambda, \theta)\end{array}$ & 55.131 & 0.0267 & 0.2097 & $\begin{array}{l}0.0575 \\
(0.9673)\end{array}$ & $\begin{array}{l}8.8699 \\
(16.810)\end{array}$ & $\begin{array}{l}112.54 \\
(627.38)\end{array}$ & $\begin{array}{l}0.3562 \\
(0.9223)\end{array}$ & $\begin{array}{l}-0.1036 \\
(4.9790)\end{array}$ \\
\hline $\begin{array}{l}\mathrm{BEx} \\
(\mathrm{a}, \mathrm{b}, \lambda)\end{array}$ & 56.177 & 0.0874 & 0.5737 & $\begin{array}{l}0.0682 \\
(0.8809)\end{array}$ & $\begin{array}{l}24.317 \\
(3.9884)\end{array}$ & $\begin{array}{l}92.491 \\
(154.90)\end{array}$ & $\begin{array}{l}0.0947 \\
(0.1426)\end{array}$ & \\
\hline $\begin{array}{l}\mathrm{Ga} \\
(\mathrm{a}, \mathrm{b})\end{array}$ & 55.165 & 0.0871 & 0.5718 & $\begin{array}{l}0.0681 \\
(0.8821)\end{array}$ & $\begin{array}{l}24.228 \\
(3.9559)\end{array}$ & $\begin{array}{l}9.7800 \\
(1.6134)\end{array}$ & & \\
\hline $\begin{array}{l}\text { TGEx } \\
(\alpha, \lambda, \theta)\end{array}$ & 59.771 & 0.1759 & 1.1229 & $\begin{array}{l}0.0843 \\
(0.6684)\end{array}$ & $\begin{array}{l}90.153 \\
(38.889)\end{array}$ & $\begin{array}{l}2.2154 \\
(0.1876)\end{array}$ & $\begin{array}{l}-0.6975 \\
(0.2062)\end{array}$ & \\
\hline $\begin{array}{l}\text { EEx } \\
(\alpha, \lambda)\end{array}$ & 60.803 & 0.2172 & 1.4053 & $\begin{array}{l}0.0953 \\
(0.5121)\end{array}$ & $\begin{array}{l}89.435 \\
(32.476)\end{array}$ & $\begin{array}{l}2.0192 \\
(0.1716)\end{array}$ & & \\
\hline $\begin{array}{l}\text { APEx } \\
(\alpha, \lambda)\end{array}$ & 75.306 & 0.1158 & 0.7520 & $\begin{array}{l}0.1919 \\
(0.0086)\end{array}$ & $\begin{array}{l}1938073 \\
(16777)\end{array}$ & $\begin{array}{l}1.2587 \\
(0.0549)\end{array}$ & & \\
\hline $\begin{array}{l}\text { Ex } \\
(\lambda)\end{array}$ & 142.129 & 0.0875 & 0.5749 & $\begin{array}{l}0.4495 \\
(0.0000)\end{array}$ & $\begin{array}{l}0.4037 \\
(0.0469)\end{array}$ & & & \\
\hline
\end{tabular}

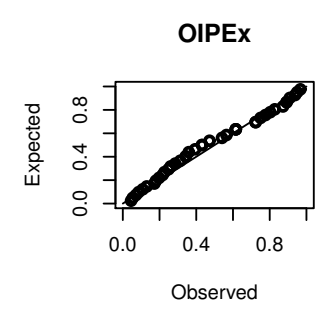

Ga

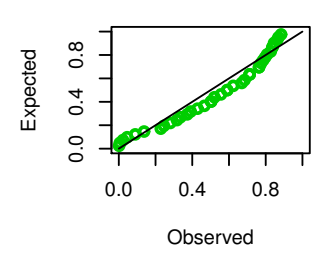

TGEx

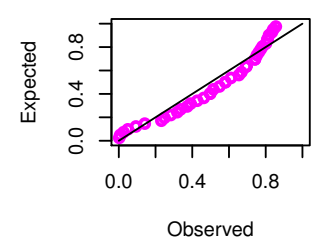

KTEx

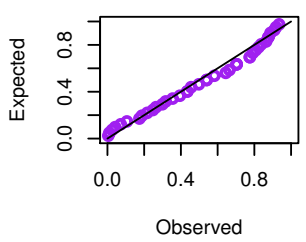

BEx

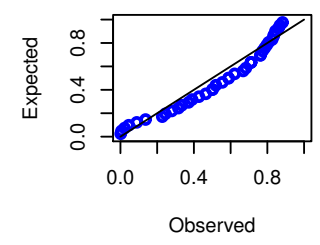

EEx

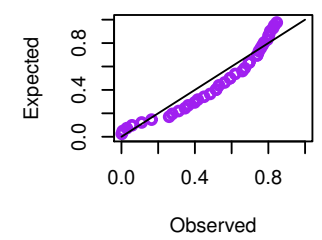

MOLEx

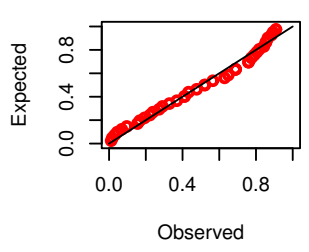

APEx

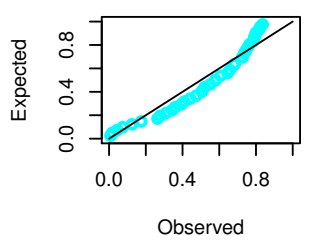

Ex

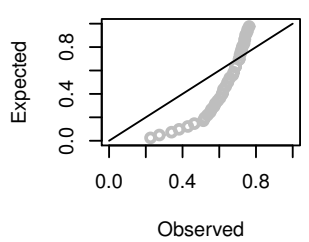

Figure 4: PP plots of the OIPEx distribution and other competitive distributions for first data set. 


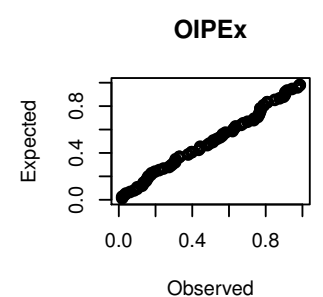

BEx

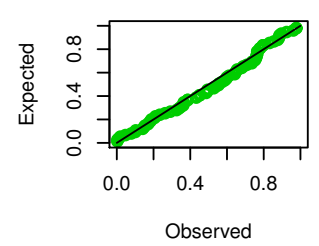

EEx

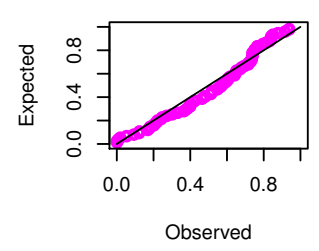

MOLEX

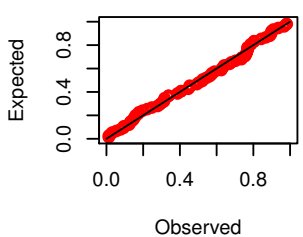

Ga

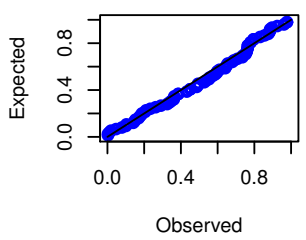

APEx

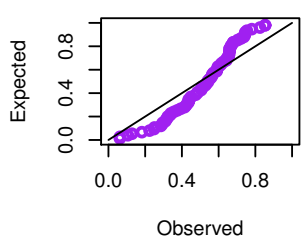

KTEX

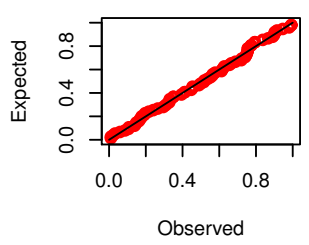

TGEX

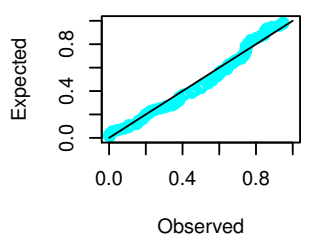

Ex

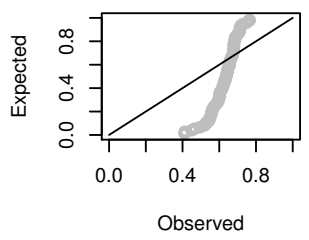

Figure 5: PP plots of the OIPEx distribution and other competitive distributions for second data set.

\section{Conclusions}

We propose a new three-parameter model called odd inverse Pareto exponential (OIPEx) distribution with two extra shape parameters. The OIPEx density function can be expressed as a linear mixture of exponential densities. We derive some of its mathematical properties including the quantile and generating functions, ordinary and incomplete moments, mean residual life, mean inactivity time and order statistics. The model parameters are estimated by the maximum likelihood estimation method. We assess the performance of the maximum likelihood estimators via a simulation study. Two applications illustrate that the new OIPEx distribution provides better fits than other competitive models.

\section{Acknowledgment}

The authors would like to thank the editor and anonymous reviewers, for their valuable constructive comments, which greatly improved the paper. This work was funded by University of Jeddah, Saudi Arabia, under grant No. (UJ-02-095-DR). The authors, therefore, acknowledge with thanks the University technical and financial support.

\section{References}

[1] A. Z. Afify, E. Altun, M. Alizadeh, G. Ozel, G. G. Hamedani, The odd exponentiated half-logistic-G family: properties, characterizations and applications, Chil. J. Stat., 8 (2017), 65-91. 5

[2] A. Z. Afify, G. M. Cordeiro, N. S. Butt, E. M. M. Ortega, A. K. Suzuki, A new lifetime model with variable shapes for the hazard rate, Braz. J. Probab. Stat., 31 (2017), 516-541. 1, 5

[3] A. Z. Afify, G. M. Cordeiro, H. M. Yousof, A. Alzaatreh, Z. M. Nofal, The Kumaraswamy transmuted-G family of distributions: properties and applications, J. Data Sci., 14 (2016), 245-270. 5

[4] A. Z. Afify, O. A. Mohamed, A new three-parameter exponential distribution with variable shapes for the hazard rate: estimation and applications, Mathematics, 8 (2020), 1-17. 1 
[5] A. Z. Afify, M. Zayed, M. Ahsanullah, The extended exponential distribution and its applications, J. Stat. Theory Appl., 17 (2018), 213-229.

[6] M. A. Aldahlan, A. Z. Afify, A. N. Ahmed, The odd inverse Pareto-G class: properties and applications, J. Nonlinear Sci. Appl., 12 (2019), 278-290. 1, 3

[7] R. C. Gupta, P. L. Gupta, R. D. Gupta, Modeling failure time data by Lehmann alternatives, Comm. Statist. Theory Methods, 27 (1998), 887-904. 1

[8] R. D. Gupta, D. Kundu, Exponentiated exponential family: an alternative to gamma and Weibull distributions, Biom. J., 43 (2001), 117-130. 1, 2, 5

[9] M. C. Jones, Families of distributions arising from distributions of order statistics, Test, 13 (2004), 1-43. 1, 5

[10] M. S. Khan, R. King, I. L. Hudson, Transmuted generalized exponential distribution: a generalization of the exponential distribution with applications to survival data, Comm. Statist. Simulation Comput., 46 (2017), 4377-4398. 5

[11] D. Kundu, M. Raqab, Estimation of $\mathrm{R}=\mathrm{P}(\mathrm{Y}<\mathrm{X})$ for three parameter Weibull distribution, Stat. Probab. Lett., 79 (2009), 1839-1846.

[12] Y. G. Lan, L. M. Leemis, The logistic-exponential survival distribution, Nav. Res. Logist., 55 (2008), $252-264.1$

[13] A. Mahdavi, D. Kundu, A new method for generating distributions with an application to exponential distribution, Comm. Statist. Theory Methods, 46 (2017), 6543-6557. 5

[14] M. Mansoor, M. H. Tahir, G. M. Cordeiro, S. B. Provost, A. Alzaatreh, The Marshall-Olkin logistic exponential distribution, Comm. Statist. Theory Methods, 48 (2019), 220-234. 5

[15] A. W. Marshall, I. Olkin, A new method for adding a parameter to a family of distributions with application to the exponential and Weibull families, Biometrika, 84 (1997), 641-652. 1, 2

[16] S. Nadarajah, F. Haghighi, An extension of the exponential distribution, Statistics, 45 (2011), 543-558. 1

[17] M. Nassar, A. Z. Afify, S. Dey, D. Kumar, A new extension of Weibull distribution: properties and different methods of estimation, J. Comput. Appl. Math., 336 (2018), 439-457. 5

[18] M. Rasekhi, M. Alizadeh, E. Altun, G. G. Hamedani, A. Z. Afify, M. Ahmad, The modified exponential distribution with applications, Pakistan J. Statist., 33 (2017), 383-398. 1

[19] K. Xu, M. Xie, L. C. Tang, S. L. Ho, Application of neural networks in forecasting engine systems reliability, Appl. Soft Compu., 2 (2003), 255-268. 5 